\title{
BASES FISIOPATOLÓGICAS DE LAS MODIFICACIONES ENDOCRINO METABÓLICAS POST-CIRUGÍA BARIÁTRICA EN DIABETES TIPO 2
}

\author{
PATHOPHYSIOLOGICAL BASIS OF ENDOCRINE METABOLIC \\ MODIFICATIONS AFTER BARIATRIC SURGERY IN TYPE 2 DIABETES
}

María Virginia Rodríguez'1, Gustavo Frechtel ${ }^{2}$

\section{RESUMEN}

Cuando la diabetes mellitus tipo 2 (DM2) se asocia a obesidad mórbida, frecuentemente la eficacia a largo plazo de las intervenciones terapéuticas no logran los objetivos ni disminuyen el riesgo cardiovascular o las complicaciones crónicas. Datos emergentes postulan a la cirugía bariátrica como tratamiento efectivo para la obesidad y sus comorbilidades.

El objetivo del presente trabajo es investigar sobre esta nueva alternativa terapéutica de la DM2 analizando desde la fisiopatología hasta los predictores de suceso y fracaso en los resultados post-quirúrgicos.

La reorganización intestinal post-quirúrgica produciría modificaciones hormonales en los ácidos biliares, sensado de nutrientes, microbioma intestinal, incretinas y anti-incretinas, asignándose un rol clave como responsable de los efectos normoglucemiantes. No obstante, la fisiología y los mecanismos moleculares subyacentes aún no se comprenden totalmente. Numerosos ensayos clínicos aleatorios a corto/mediano plazo demuestran que la cirugía metabólica logra un excelente control glucémico y reduce factores de riesgo cardiovascular. Aunque se necesitan estudios adicionales para demostrar beneficios a largo plazo, existe suficiente evidencia que apoya su inclusión entre las intervenciones contra la DM2. Una mejor comprensión de esta constelación de factores que intervienen en la homeostasis glucémica abre el camino hacia potenciales dianas terapéuticas, incluyendo drogas específicas, técnicas menos invasivas, optimización de los resultados quirúrgicos y fundamentalmente promover una mayor individualización de la terapia en la DM2.

Palabras claves: cirugía bariátrica, obesidad, derivación gástri$\mathrm{ca}$, diabetes mellitus.

Revista de la Sociedad Argentina de Diabetes 2016; Vol. 50 (108-116)

\section{ABSTRACT}

The long-term efficacy of therapeutic interventions have often failed to achieve the objectives and reduction of cardiovascular risk or chronic complications when diabetes mellitus type 2 (DM2) is associated with morbid obesity. Emerging data postulate bariatric surgery as an effective treatment for obesity and its comorbidities.

The aim of this study is to conduct a research about this new DM2 therapeutic alternative, analyzing from the pathophysiology until the predictors of success and failure of postsurgical results.

Postsurgical gut reorganization generates hormonal changes, modifications in bile acids, nutrient sensing, incretins, antiincretinas and gut microbioma; whose role is key since they are responsible for the effects in glucose homeostasis. However, the physiology and molecular mechanisms underlying are not still fully understood. Numerous short and medium term randomized clinical trials have proved that through metabolic surgery an excellent glycemic control can be achieved thus reducing cardiovascular risk factors and mortality. Although further studies are needed to demonstrate long-term benefits there is enough evidence supporting their inclusion among interventions against DM2.

A better understanding of this constellation of factors involved in glucose homeostasis opens the way to potential therapeutic targets including specific drugs, less invasive techniques, optimization of surgical results and fundamentally promote greater individualization of DM2 therapy.

Key words: bariatric surgery, obesity, gastric bypass, diabetes mellitus.

Revista de la Sociedad Argentina de Diabetes 2016; Vol. 50 (108-116)
Instituto Centenario, CABA, Argentina

2 Prof. Asociado de la Cátedra de Nutrición, Facultad de Medicina, UBA, Jefe del Servicio de Nutrición y Diabetes, Hospital Sirio Libanés, Jefe de la División Genética, Hospital de Clínicas, CABA, Argentina
Contacto del autor: María Virginia Rodríguez

E-mail: dramariavirginiarodriguez@gmail.com

Correspondencia: Junín 1631,70 "C" (C1113AAK), CABA,

Argentina

Tel.: (0351) 152661889

Fecha de trabajo recibido: 15/10/16

Fecha de trabajo aceptado: 14/12/16

Conflictos de interés: los autores declaran no presentar conflictos de interés 


\section{INTRODUCCIÓN}

La Federación Internacional de Diabetes (IDF) reportó que 366 millones de personas presentaban diabetes en el año 2011 en el mundo y se estimó que estarán afectadas aproximadamente $552 \mathrm{mi}-$ llones de personas para el año 2030'. Las intervenciones terapéuticas con dietas hipocalóricas, ejercicio físico y fármacos mejoran la fisiopatología de la diabetes mellitus tipo 2 (DM2), preservan la función de la célula $\beta$ y representan el tratamiento de primera línea para estos pacientes ${ }^{2}$. No obstante, la eficacia a largo plazo de estas intervenciones en algunos pacientes es pobre, no logra llegar a los objetivos terapéuticos ni disminuir el riesgo cardiovascular y las complicaciones crónicas de la enfermedad. Cuando la DM2 se asocia a obesidad mórbida, su control se torna más complejo dado que algunos medicamentos y la intensificación del tratamiento suelen acentuar y acelerar el aumento del peso ${ }^{3}$. Existen datos emergentes que sugieren que la cirugía bariátrica podría proveer un tratamiento más efectivo y sostenido para la obesidad y sus morbilidades asociadas ${ }^{4,5}$. La "cirugía metabólica" ha sido incorporada como parte del tratamiento en las guías de las sociedades de diabetes incluida la Asociación Americana de Diabetes (ADA) y la Federación Internacional de Diabetes (FID)6.

El objetivo del presente trabajo es realizar una investigación sobre esta nueva alternativa terapéutica, analizar desde la fisiopatología hasta los predictores de suceso y fracaso, y comprender los mecanismos de remisión y recaída de la DM2.

\section{Diabetes mellitus 2}

La DM2 es una enfermedad hereditaria poligénica que resulta de la interacción de múltiples variantes genéticas en distintos genes y su interacción con factores medioambientales. Tiene múltiples etiologías caracterizadas por una combinación de insulinorresistencia y un defecto en la función de la célula $\beta$ que empeora con el tiempo y provoca un defecto en el metabolismo de los hidratos de carbono, lípidos y proteínas? ${ }^{7}$. La insulinorresistencia muscular y hepática junto con la falla de la célula $\beta$ representan los principales defectos fisiopatológicos. En conjunto con estos factores, la célula adiposa (lipólisis acelerada), el tracto gastrointestinal (deficiencia/resistencia incretínica), el riñón (incremento en la reabsorción de glucosa) y el cerebro (insulinorresistencia) juegan un rol importante en el desarrollo de intolerancia a la glucosa ${ }^{8}$. Las anorma- lidades del eje incretínico intestinal también demostraron jugar un rol importante en la falla progresiva de la célula $\beta$. Por otro lado, la microbiota intestinal alterada se asocia con enfermedades crónicas que incluyen obesidad y $\mathrm{DM}^{9}{ }^{9}$. En cuanto a la regulación del apetito, el estómago produce ghrelina que regula la actividad en aéreas del SNC implicadas en la recompensa y la regulación homeostática de las reservas energéticas a largo plazo, así como en el hipotálamo y en el núcleo accumbens ${ }^{10}$.

El manejo de la glucemia en la DM2 se ha vuelto cada vez más complejo dado que se encuentra disponible una serie cada vez más extensa de agentes farmacológicos, mayores preocupaciones sobre sus efectos adversos potenciales y nuevas incertidumbres respecto de los beneficios del control glucémico intensivo sobre las complicaciones macrovasculares ${ }^{11}$. El conocimiento de la fisiopatología de esta enfermedad tiene importantes implicaciones terapéuticas: 1) el tratamiento efectivo de la DM2 requerirá el uso combinado de múltiples drogas para corregir sus variadas alteraciones fisiopatológicas; 2) el tratamiento debe dirigirse a las anormalidades fisiopatológicas de las comorbilidades como obesidad, dislipemia aterogénica, hipertensión arterial y no sólo a la reducción de hemoglobina glicosilada (HbA1c); 3) la terapia debe comenzar temprano en la historia natural de la DM2 para prevenir el deterioro progresivo de la célula $\beta$ y las complicaciones micro y macrovasculares ${ }^{12}$; 4) la individualización del tratamiento es la piedra angular del éxito en la $\mathrm{DM}^{4,11}$.

El manejo de la DM2 consiste en lograr un estilo de vida saludable con dieta, ejercicio y educación como base de cualquier programa de tratamiento. Sin embargo muchos pacientes no logran reducir el peso corporal ni alcanzan los objetivos terapéuticos. En el UKPDS (United Kindom Prospective Study) se observó que los pacientes con sobrepeso y diabetes recién diagnosticados aumentan aproximadamente entre 2 y $7 \mathrm{~kg}$ durante 10,7 años de seguimiento ${ }^{13}$. En el Steno-2 en pacientes con DM2 y albuminuria se observó un aumento progresivo tanto del IMC como de la circunferencia de cintura durante 13,3 años de seguimiento ${ }^{14}$. Debido a la disfunción progresiva de la célula $\beta$, característica de esta enfermedad crónica, la monoterapia deja de ser efectiva con el tiempo y se impone la necesidad de agregar una segunda línea farmacológica o insulina. Las evidencias demuestran el poder de las intervenciones del estilo de vida para prevenir el desarrollo 
de DM2 en poblaciones de riesgo, pero los individuos con DM2 pueden tener más dificultades para lograr y sostener una pérdida de peso a largo plazo, tanto por el deterioro progresivo de la célula $\beta$ como por el escaso cumplimiento y adherencia de las medidas dietéticas como farmacológicas ${ }^{15}$.

\section{Cirugía bariátrica como herramienta tera- péutica en la diabetes y sus comorbilidades}

\section{Mecanismos metabólicos y hormonales que revierten la DM2 luego de la cirugía bariátrica}

Existen controversias sobre los mecanismos que conducen a la mejoría metabólica luego de la cirugía bariátrica en los pacientes con DM2. Muchas se fundamentaron en la recuperación de la sensibilidad hepática a la glucosa por la restricción energética, aunque actualmente creció la aceptación sobre los cambios en las hormonas enterales, adaptaciones neuronales y plasticidad intestinal como consecuencia de los cambios anatómicos del BGYR.

La Tabla 1 muestra el conjunto de mecanismos propuestos mediante el cual se hipotetiza que la cirugía bariátrica mejora los mecanismos fisiopatológicos de la DM2.

\section{a. Cambios en la insulinosensibilidad posterior a cirugía bariátrica}

El índice de insulinosensibilidad mediante el test de tolerancia endovenosa a la glucosa se incrementó entre 4-5 veces. Hay aumento de las concentraciones de receptores de insulina y marcadores de señalización intracelular musculares, así como también la expresión del cofactor de transcripción mitocondrial PPAR 'Y- coactivador 1 (PGC-1) y su target la mitofusina 2. Ambos estimulan el metabolismo de ácidos grasos y predicen la reducción de lípidos intramiofibrilares ${ }^{16}$. El rango de remisión intrahospitalaria de la diabetes se ha reportado hasta en un $89 \%$. En contraste, las cirugías restrictivas puras como la gastrectomía vertical en manga (GVM) mejoran el metabolismo glucídico sólo después de que se ha logrado una pérdida de peso substancial ${ }^{17}$. Por este motivo se investigaron líneas de evidencias que plantean mecanismos independientes del descenso de peso que mejorarían el control de la diabetes posterior a la cirugía metabólica.

\section{b. Mecanismos hormonales}

- Ghrelina. Más del 90\% es producida en el estómago y duodeno, ambos afectados por el BGYR por lo cual la regulación metabólica producida por ghrelina estaría alterada después de la cirugía. Este hecho proveería una base posible para reducir el craving alimentario (picoteo compulsivo), así como la mejoría de glucemia ${ }^{18}$.

- Péptidos intestinales. Los cambios del patrón de secreción de hormonas intestinales por las células enteroendócrinas en respuesta a la modificación del tránsito de nutrientes post-quirúrgico se considera un mecanismo potencial de mejoría de la DM2. Las células I y $\mathrm{K}$ del intestino proximal producen principalmente colecistoquinina (CCK) y péptido insulinotrópico estimulado por glucosa (GIP) respectivamente, mientras que las células $L$ del intestino distal producen GLP-1, GLP-2, oxintomodulina y péptido YY (PYY), el conjunto de los cuales contribuye a la saciedad ${ }^{18}$.

- Incretinas y péptido YY. En el postprandial, tanto del BPYR como en la GVM, los niveles de GLP-1 están dramáticamente incrementados ${ }^{18}$, sin embargo este efecto no se observa en las cirugías restrictivas ni en la pérdida de peso secundaria a dieta $^{19}$. La recuperación temprana en la función de la célula $\beta$ se ha asociado con una secreción postprandial exagerada de GLP1 en el post-quirúrgico. Los estudios de Martinussen et al. demostraron recuperación de la primera fase de secreción de insulina y mejoría del HOMA- $\beta$ en pacientes con DM2 dentro de la primera semana post BGYR. A largo plazo, el GLP-1 posiblemente podría estimular la regeneración e hipertrofia de célula $\beta$ e incrementaría además la señal a nivel vagal ${ }^{20}$.

- Glucagón y GLP-2. La glucagonemia de ayunas decrece después de un año post-quirúrgico y podría explicar algunos de los beneficios a largo plazo en la homeostasis glucídica ${ }^{21}$. Le Roux et al. encontraron que los niveles de GLP-2 crecieron con un pico entre los seis a 12 meses post BGYR, correlacionándose con los períodos de mayor pérdida de peso; esto se asoció con una mayor proliferación de las criptas como mecanismo de adaptación en la anatomía intestinal para minimizar la malabsorción de macronutrientes ${ }^{22}$.

- Tejido adiposo y adipokinas. Luego del BGYR se muestra un incremento de adiponectina y reducción de las concentraciones de visfatina, leptina, TNF- $\alpha$, IL-6, PCR y de la infiltración de macrófagos en el tejido adiposo ${ }^{23}$.

\section{c. Mecanismos intestinales}

- Hipótesis del intestino distal. La llegada tem- 
prana y acelerada de nutrientes no digeridos al intestino delgado distal activaría el "freno intestinal" que potenciaría la secreción de GLP-1 y PYY. Consecuentemente se observa cómo los procedimientos con mayor mejoría de la DM2 son aquellos que acortan la ruta del flujo de nutrientes desde el estómago al intestino y/o incrementan la tasa de transporte de los nutrientes ingeridos ${ }^{24}$. Una posibilidad planteada es que la activación de las células $L$ sea una respuesta de emergencia ante los altos niveles de vaciamiento gástrico, y el incremento de GLP-1 y PYY sería un mecanismo inefectivo para reducir este vaciamiento gástrico acelerado ${ }^{25}$.

- Hipótesis del intestino proximal. Plantea que la exclusión del intestino proximal (principalmente del duodeno) del contacto con los nutrientes ejercería efectos antidiabéticos directos, presumiblemente vía uno o más factores duodenales no identificados ("anti-incretinas") que influirían en la homeostasis de la glucosa. Una candidata fuerte fue recientemente descripta por Alfa et al. nombrando decretina a una proteína descubierta en drosophila (limostatin), la cual suprimía la producción y liberación de insulina ${ }^{26}$. En humanos se demostraron reducciones de la $\mathrm{HbA} 1 \mathrm{c}$ luego del implante de varios tipos de endoprótesis endoluminales que realizan un bypass duodenoyeyunal, sugiriendo una terapia noble y menos invasiva para el tratamiento de la DM2 ${ }^{27 .}$

- Enteroplasticidad

- Adaptación en la morfología: después del BGYR el asa alimentaria experimenta hiperplasia e hipertrofia, junto con el aumento de la expresión de transportadores de glucosa, incrementando el ingreso de glucosa a las células del epitelio intestinal y reprogramando el metabolismo glucémico intestinal para sostener el crecimiento tisular y las demandas energéticas aumentadas. También se incrementa el número de células productoras de GLP-1 y GIP28.

- Cambios en el sistema nervioso central: el BGYR induce alteraciones en la inervación vagal similares a la vagotomía troncal subdiafragmática que podrían reflejarse por una reorganización a largo plazo de los centros de alimentación del cerebro posterior: núcleo del tracto solitario (NTS) y el núcleo motor dorsal del vago (NDV) y una disminución de la señal vagal, así como de glutamato en el NTS. Esta señal disminuiría la ingesta y reduciría el peso corporal ${ }^{25}$.

- Cambios entero-endocrinos: se demostró la expresión de enzimas clave de la gluconeogénesis intestinal inducidas en estado de déficit ener- gético; la producción de glucosa resultante activaría los sensores portales de glucosa (GLUT 2) para estimular un neurocircuito que incrementaría la insulinosensibilidad hepática al disminuir la liberación de glucosa (eje intestino-cerebro-hígado) ${ }^{25,29}$.

- Censado de nutrientes: en la cirugía bariátrica, el asa común es la que se encuentra más afectada en cuanto al aumento de las demandas metabólicas causadas por el vaciamiento rápido del estómago que altera la presentación de nutrientes, las fuerzas intraluminares y produce cambios dramáticos en el ph. Los receptores de gusto intestinales (TAS1R2) acoplados a la proteína G detectan los contenidos luminares y transmiten señales que regulan la expresión de los transportadores de nutrientes y el ingreso de los mismos, así como la liberación de hormonas intestinales y neurotransmisores relacionados con la regulación de la energía y la homeostasis de glucosa ${ }^{25}$. La regulación de los TAS1R2 se encuentra alterada en la DM2 predisponiendo a hiperglucemia postprandial por incremento de la absorción de glucosa por el cotransportador Na+/glucosa (SGLT1) durante la hiperglucemia. La llegada acelerada de nutrientes no digeridos al intestino delgado distal después de BGYR podría afectar también la regulación de los receptores del gusto y los transportadores de glucosa en las células $L$ llevando al incremento de PYY, GLP-1 y de la expresión de TAS1R3 en el limbo alimentario y la disminución del transportador SGLT1. Esto provocaría la disminución de la preferencia por lo dulce y las comidas ricas en grasa que se manifiesta en los pacientes post BGYR conduciendo a alteraciones en el circuito de recompensa y homeostasis de energía ${ }^{30}$.

- Ácidos biliares (AB): los niveles de AB circulantes se incrementan en el postoperatorio, correlacionándose con la mejoría de la tolerancia a la glucosa. El reordenamiento anatómico postquirúrgico conduciría a retrasar la mezcla de los $A B$ con los alimentos y la exposición al íleo del quimo no digerido libre ofreciendo una explicación posible para este fenómeno ${ }^{31}$. Los $A B$, además, se comportan como hormonas sobre receptores nucleares y de membrana en las células del hígado e intestino. Existen transportadores y receptores de $A B$ en intestino, hígado, riñón y corazón, lo cual sugiere un rol global de señalización fuera del circuito enterohepático ${ }^{32}$. Se identificaron dos receptores: a) TGR5, receptor 1 de ácidos biliares acoplado a proteína $\mathrm{G}$ en la superficie celular en el 
intestino: incrementan el gasto energético a nivel del tejido adiposo pardo mediante la activación de la dehiodinasa tiroidea tipo 2 (convierte T4 en T3), y en las células $L$ inducen la secreción de GLP-1; b) FXR, receptor factor $X$ farnesoide: estimula la secreción del FGF-19 (factor de crecimiento fibroblástico) regulador de la producción de $A B$ con la consecuente inhibición de la gluconeogénesis hepática; la señal del FXR sería clave además en los cambios de la microbiota dado que ratones operados de BGYR y que son knock out para el FXR no muestran una microbiota del fenotipo delgado ${ }^{33}$.

- Microbiota: posterior al BGYR se observaron modificaciones en la composición bacteriana a nivel de phylum, disminuyendo Firmicutes y aumentando Bacteroidetes, junto con una extensión de la diversidad bacteriana. Otro hallazgo es el aumento de Gammaproteobacteria (Escherichia) y Verrucomicrobia (Akkermansia), que correlacionan con el descenso de peso y la mejoría de factores inflamatorios. En DM2 con BGYR se observa aumento del Faecalobacterium prausnitzii (Firmicutes) asociado a mejoría de la insulinorresistencia y disminución de IL-6 y PCRus ${ }^{34}$. Liou et al. demostraron que el trasplante de materia fecal de roedores con BGYR a animales germ free producía pérdida de peso y masa grasa en los receptores en comparación con los receptores de microbiota de ratas con operación simulada que ganaron peso ${ }^{35}$.

\section{d. Inflamación}

Estudios recientes encontraron una reducción significativa de la endotoxemia y mediadores proinflamatorios después de la cirugía bariátrica en diabéticos y obesos mórbidos tanto a los seis meses como al año. En concordancia con la modificación de la microbiota intestinal, esto podría influir en los niveles circulantes de LPS $36,37$.

\begin{tabular}{|c|c|c|c|}
\hline Fisiopatología DM2 & Impacto inmediato de la CX & Mediadores potenciales & $\begin{array}{c}\text { Efectos de la homeostasis } \\
\text { de la glucosa }\end{array}$ \\
\hline $\begin{array}{l}\text { Célula } \beta \\
\downarrow \text { de la secreción de insulina }\end{array}$ & Restricción calórica & Pérdida de peso & $\begin{array}{l}\text { Mejoría de la función de } \\
\text { célula } \beta \text { y masa celular }\end{array}$ \\
\hline $\begin{array}{l}\text { Célula } \alpha \\
\uparrow \text { de la secreción de glucagón }\end{array}$ & $\downarrow$ glucagonemia & $\begin{array}{l}\text { Alteración de las } \\
\text { hormonas intestinales } \\
\text { (incretinas, péptido YY) }\end{array}$ & $\begin{array}{l}\text { Reducción de } \\
\text { glucotoxicidad y } \\
\text { lipotoxicidad }\end{array}$ \\
\hline \multirow[t]{2}{*}{$\begin{array}{l}\text { Intestino } \\
\downarrow \text { del efecto incretina }\end{array}$} & Hipótesis intestino distal & $\begin{array}{c}\text { *Freno intestinal* } \\
\text { Censado de nutrientes } \\
\text { (TAS1 R3) }\end{array}$ & \multirow{2}{*}{$\begin{array}{l}\text { Cambios en el } \\
\text { circuito de } \\
\text { recompensa y de } \\
\text { homeostasis de } \\
\text { energía }\end{array}$} \\
\hline & $\begin{array}{l}\text { Hipótesis de intestino } \\
\text { proximal }\end{array}$ & $\begin{array}{l}\text { ¿Anti-incretinas? Drosophila } \\
\text { Limostatin (Lst). }\end{array}$ & \\
\hline \multirow[t]{2}{*}{$\begin{array}{l}\text { Hígado } \\
\uparrow \text { de producción hepática } \\
\text { de glucosa }\end{array}$} & Cambios enteroendocrinos & \multirow[t]{2}{*}{$\begin{array}{l}\text { Adaptaciones morfológicas. } \\
\text { Alteración de la señalización } \\
\text { de AB: TGR5/FGF-19 y FXR. }\end{array}$} & \multirow{2}{*}{$\begin{array}{c}\text { Mejoría de } \\
\text { insulinosensiblidad } \\
\text { Inhibición de la } \\
\text { gluconeogénesis hepática }\end{array}$} \\
\hline & Enteroplasticidad & & \\
\hline $\begin{array}{l}\text { Músculo } \\
\downarrow \text { la captación muscular de glucosa }\end{array}$ & $\begin{array}{l}\text { Cambios en } \\
\text { insulinosensibilidad }\end{array}$ & $\begin{array}{c}\uparrow \text { cofactor de transcripción } \\
\text { mitocondrial PGC-1 y su target } \\
\text { la mitofusina } 2\end{array}$ & \multirow[t]{2}{*}{$\begin{array}{l}\text { Incremento en la } \\
\text { utilización de } \\
\text { glucosa y AG }\end{array}$} \\
\hline $\begin{array}{l}\text { Adipocito } \\
\uparrow \text { adipoquinas proinflamatorias } \\
\uparrow \text { Lipólisis }\end{array}$ & Mecanismos inflamatorios & $\begin{array}{c}\uparrow \text { adiponectina, } \downarrow \text { adipokinas } \\
\text { proinflamatorias } \\
\downarrow \text { LPS, AGCC }\end{array}$ & \\
\hline $\begin{array}{l}\text { Cerebro } \\
\text { Disfunción de neurotransmisores }\end{array}$ & $\begin{array}{l}\text { Cambios en la inervación } \\
\text { vagal, actividad neuronal } \\
\text { Remoción del fundus } \\
\text { estomacal ( } \downarrow \text { ghrelina) }\end{array}$ & $\begin{array}{c}\text { Reorganización de centros } \\
\text { de alimentación de cerebro } \\
\text { posterior (NTS y NDV) }\end{array}$ & Disminución de la ingesta \\
\hline \multicolumn{4}{|l|}{ Riñón $\uparrow$ reabsorción de glucosa renal } \\
\hline Genética y epigenética & $\begin{array}{l}\text { Influencia genética y } \\
\text { mecanismos epigenéticos }\end{array}$ & $\begin{array}{l}\text { FTO } \\
\text { TCF7L2 } \\
\text { FOXO } 1\end{array}$ & $\begin{array}{l}\text { Factores asociados a } \\
\text { remisión de diabetes } \\
\text { post-quirúrgica }\end{array}$ \\
\hline
\end{tabular}

Tabla 1: Mecanismos propuestos en la mejoría del metabolismo glucémico por la cirugía metabólica. Adaptado de: Batterham R, et al. Mechanisms of Diabetes improvement following bariatric/metabolic surgery ${ }^{37}$. 


\section{Control glucémico post-cirugía bariátrica}

\section{Evidencia referente a la remisión de la diabetes post-cirugía bariátrica}

Con respecto a la definición de resolución de diabetes, se tomaron los criterios del Consenso de Cirugía Metabólica publicados en la revista de la SAD ${ }^{38,39}$.

En el trabajo de Mingrone et al. la comparación del tratamiento quirúrgico vs el mejor tratamiento médico en el control metabólico presentó mejores resultados para el quirúrgico ${ }^{4}$. Estudios observacionales de procedimientos bariátricos han demostrado rangos de remisión de DM2 de 55 a 95\% ${ }^{40}$. Un metaanálisis del año 2013 de estudios randomizados controlados mostró una remisión de diabetes 22 veces mayor con tratamiento quirúrgico que con el convencional ${ }^{41}$.

En el estudio STAMPEDE que evaluó la eficacia del tratamiento médico solo o combinado con BGYR o GVM para alcanzar criterio de remisión de DM2, después de un año se alcanzó la remisión en el $42 \%$ de los sometidos a BGYR, $37 \%$ en GVM y $12 \%$ con terapia convencional. La inclusión de pacientes con DM2 más avanzada en este estudio probablemente explicaría el menor rango de remisión de $\mathrm{DM}^{42}$.

El estudio SOS (Swedish Obese Subjects) demostró remisión de DM2 a dos años post-quirúrgico del $72 \%$ vs $16,4 \%$ en tratamiento convencional y a 10 años de $30,4 \%$ vs $13,3 \%$ respectivamente (remisión prolongada $)^{43}$. A pesar de la declinación en la remisión de DM2 a los 10 años, permaneció la reducción significativa en eventos macro y microvasculares en comparación con el grupo control. Otra revisión de estudios a cinco años detectó que el $24 \%$ de los pacientes logró remisión completa y otro 16\% remisión parcial. De aquellos pacientes que inicialmente remitieron, 19\% presentó recurrencia de su diabetes a largo plazo. Sin embargo, los pacientes disminuyeron el número de medicamentos y pocos requirieron insulina en comparación con el pre-quirúrgico ${ }^{44}$.

La técnica quirúrgica también influye sobre la eficacia del tratamiento y la remisión. En las intervenciones restrictivas la mejoría depende principalmente del descenso de peso, mientras que en las malabsortivas se produciría por mecanismos independientes del peso ${ }^{45}$. Según una revisión reciente, los procedimientos como BGYR y DBP producen un 80 y $95 \%$ de remisión de la hiperglucemia respectivamente, un efecto notablemente mayor que el observado en la mayoría de los estudios convencionales de dieta y terapia farmacológica ${ }^{46}$.
Publicaciones sobre roedores han demostrado que el BYD mejora la diabetes tanto en ratas obesas (Zucker ob/ob) como en delgadas (Goto-Kakizaki); en ratas no obesas con DM2 esta operación mejoró de manera rápida y duradera la DM2, incluso sin causar reducción de la ingesta de comida o peso corporal en comparación con los controles ${ }^{47}$. Esto es coincidente con las observaciones de remisión de diabetes con las cirugías de BPYR o DBP48.

La cirugía también previene nuevos casos de diabetes. En el estudio SOS a los 10 y 15 años la incidencia de DM2 disminuyó un 96 y 78\% respectivamente, con lo que se alcanzó aproximadamente el doble de resultados favorables comparado con los logrados en programas de cambios intensivos en el estilo de vida. Por el contrario, el IMC no predice el efecto preventivo de la cirugía bariátrica sobre la $\mathrm{DM}^{249,50}$.

En el estudio de extensión del STAMPEDE a los 24 meses la proporción de pacientes con $\mathrm{HbA} 1 \mathrm{c}<6 \%$ persistió en el grupo con BGYR, al igual que el TTOG con una disminución marcada de los niveles de glucosa en ayunas y postprandiales. Los niveles de péptido $C$ postprandiales y la velocidad de secreción de insulina a los 30 y 60 minutos aumentaron más de dos veces en el BGYR y la GVM. Estos efectos se asociaron con un aumento de 5,8 veces la función total de las células $\beta$. En los pacientes con BGYR aumentaron tanto la sensibilidad a la insulina como los componentes de secreción, pero en la gastrectomía en manga, pese a una pérdida de peso similar, la sensibilidad a la insulina sólo se recuperó parcialmente y la función de las células $\beta$ no mejoró 51 .

\section{Predictores de remisión de DM2}

Los factores asociados con menor rango de remisión y/o mayor rango de recidiva post-quirúrgica son: una diabetes de larga evolución (8 a 10 años), con mayor requerimiento de insulina previo a la cirugía y un pobre control glucémico ${ }^{52}$. El mayor porcentaje de grasa visceral también ayudaría a predecir una menor remisión independientemente del IMC ${ }^{44}$. Asimismo este procedimiento no produce un control similar de la glucemia en pacientes con DM1, por consiguiente se deberían solicitar anticuerpos y péptido $C$ previo a la cirugía. Estos hallazgos sugieren que el estado terminal de falla de célula $\beta$, característica de la DM de larga evolución, podría forjar la condición irreversible en estos pacientes. Consecuentemente debería ofrecerse un abordaje quirúrgico temprano por encima de última instancia en el curso natural de la enfermedad ${ }^{52}$. 


\section{Eficacia de la cirugía bariátrica en la resolu- ción de las comorbilidades asociadas a DM2}

- Obesidad. Se considera éxito en la cirugía la pérdida del $50 \%$ del exceso de peso corporal $(\mathrm{PSP})^{46}$. Según Buchwald et al. comparativamente la pérdida de peso media post BGA fue del $47,5 \%$, post BPGYR de $68,2 \%$ y 70,1\% en la derivación $\mathrm{DBP}^{51}$, mientras que con la GVM a dos años fue de $61,5 \%{ }^{53}$. El estudio de Kehagias et al. reportó un resultado similar en cuanto PSP a tres años comparando BPGYR y GVM $68 \%$ vs $62 \%$ de PSP respectivamente $)^{54}$. A pesar de esto, en la gran mayoría de los estudios comparativos la pérdida de peso y resolución de comorbilidades con GVM son inferiores a los derivados del BPGYR y el BPD ${ }^{55}$.

La mayor pérdida de peso se alcanza típicamente a los 12-18 meses post-quirúrgicos, no obstante las evidencias sugieren que algunos pacientes recuperan peso varios años después. En el SOS con seguimiento a 10 años, el PSP con BGA a dos años fue del $20 \%$, superior a lo conseguido con el tratamiento convencional pero menor que con las demás técnicas. Desde el segundo año, hubo una ganancia progresiva de peso, por lo que a los 10 años el PSP fue del 14\% y a los 15 años del 13\%. Los pacientes con BPGYR lograron un $32 \%$ de PSP a dos años; a los 10 años hubo una reganancia de peso. Sin embargo, a los 15 años el PSP total fue $27 \%{ }^{43}$.

De estos datos se extrae que tanto el BGYR como otras cirugías bariátricas raramente regresan a los pacientes a una condición completamente normal. Al perder el 50-60\% del exceso de peso significa que en varios el restante $40-50 \%$ de exceso de peso no es eliminado por la cirugía. Técnicamente muchos pacientes fallan en alcanzar la remisión completa de la obesidad. Esto es un contraste notable con la evidencia de que el $80 \%$ de los pacientes que se somete al BGYR y el $90 \%$ de los sometidos a DBP experimentan una completa remisión de la DM2. Por lo tanto, si consideramos sólo en términos de producir remisión de la enfermedad, BGYR ly la DBP) es más efectivo en el tratamiento de la diabetes que en la obesidad en sí misma ${ }^{52}$.

- Hipertensión arterial. El efecto de BGYR en la hipertensión arterial es aún poco claro. En el estudio SOS, el BPYR mostró una disminución del 4,7\% y del 10,4\% en la TA sistólica y diastólica respectivamente, pero reportó una recaída a los seis meses ${ }^{50}$. Brethauer et al. observaron un $49 \%$ de control de la HTA a los dos años post-quirúrgicos y un $62 \%$ a los cinco años. En el estudio LABS-2, la HTA remitió a los tres años en un $38 \%{ }^{56}$. En el Utah Obesity, con un seguimiento a seis años, la TAS y TAD disminuyeron en el grupo de BGYR en $8 \mathrm{mmHg}$ y 5 $m m H g$ respectivamente ${ }^{57}$. Sin embargo, Mingrone et al., a dos años, encontraron no superioridad del BGYR vs tratamiento convencional ${ }^{4}$. Consiguientemente si la cirugía bariátrica mejora la HTA continúa siendo cuestionable y requiere frecuente monitoreo y estudios de seguimiento a largo plazo.

- Dislipemia. En humanos y en ratas el BGYR rápidamente revierte la disfunción de HDL y restaura las propiedades protectoras del endotelio, incluyendo la activación de eNOS (oxido nítrico sintetasa constitutiva) y la producción de ON (óxido nítrico), así como también los efectos antioxidantes y antiapoptóticos. Además restaura la capacidad de eflujo mediado por el colesterol HDL ${ }^{58}$. En el estudio SOS, en los individuos con BGYR hubo una disminución del $28 \%$ en triglicéridos y del $12 \%$ en el colesterol total a 10 años $^{49}$. Brethauer et al. demostraron la posibilidad de alcanzar el control de los niveles de LDL y triglicéridos dentro de los dos primeros años post-quirúrgicos, que incluso se mantiene a los cinco años, mientras que el porcentaje de HDL en objetivo incrementa a largo plazo (Tabla 2$)^{59}$. Se observó también una reducción significativa ( $25 \%$ ) en las concentraciones de LDL oxidadas un año post BGYR con una reducción de un $30 \%$ de las concentraciones de LDL y VLDL. El estudio LABS-2 reportó la mejoría de la dislipemia tres años posterior al BGYR en un $62 \%$ de los individuos. El BGYR se muestra más eficiente en mejorar niveles de HDL que otros modelos de cirugía bariátrica ${ }^{56}$.

Por otro lado, la cirugía bariátrica evidenció reducir la mortalidad y en particular la mortalidad cardiovascular. En el SOS la mortalidad por todas las causas se redujo en un $23 \%$ en los pacientes con cirugía bariátrica en comparación con el tratamiento convencional ${ }^{49}$.

\begin{tabular}{|l|c|c|}
\hline Parámetro metabólico & $\begin{array}{c}\text { Corto plazo } \\
\text { (2 años) }\end{array}$ & $\begin{array}{c}\text { Largo plazo } \\
\text { (5 años) }\end{array}$ \\
\hline Presión arterial <130/80 $\mathbf{~ m m H g}$ & $49 \%$ & $62 \%$ \\
\hline LDL <100 $\mathbf{~ m g / d L ~}$ & $71 \%$ & $72 \%$ \\
\hline $\begin{array}{l}\text { HDL }>\mathbf{5 0} \mathbf{~ m g / d L} \\
\text { en } \mathbf{m u j e r e s}>\mathbf{4 0} \mathbf{~ m g / d L ~ e n ~ h o m b r e s ~}\end{array}$ & $68 \%$ & $73 \%$ \\
\hline Triglicéridos <150mg/dL & $80 \%$ & $80 \%$ \\
\hline
\end{tabular}

Tabla 2: Mejoría del perfil metabólico a corto y largo plazo de los pacientes diabéticos post-cirugía bariátrica. Adaptado de: Brethauer et al. Can diabetes be surgically cured? Long term metabolic rffects of bariatric surgery in obese patients with type 2 diabetes mellitus. 


\section{CONCLUSIONES}

La obesidad y la diabetes han irrumpido en nuestra sociedad y en el escenario quirúrgico con el impacto de una revolución, promoviendo el estudio de la fisiología del metabolismo, del control del ciclo apetito-saciedad y del intestino como órgano endocrino clave en la regulación de la glucemia. Esto ha permitido comprender mejor la fisiopatología de la diabetes y el desarrollo de sus comorbilidades al impulsar el desarrollo de nuevas herramientas para el manejo de esta enfermedad multifactorial que no ha logrado alcanzar los objetivos terapéuticos a pesar del amplio rango de intervenciones implementadas.

La cirugía bariátrica produce marcadas modificaciones hormonales que contribuyen a la saciedad y mejoría en la regulación de la glucosa, sin embargo datos recientes han movido el pasado tradicional asignando un rol clave a la señalización mediada por ácidos biliares y a las alteraciones en la comunidad microbiana intestinal (eslabón entre la dieta rica en grasas, inflamación sistémica y homeostasis de la glucosa) como contribuyentes a los potentes efectos de estas cirugías. De esta manera, estos datos sugieren un concepto noble y revolucionario acerca de una antigua enfermedad: la DM2, que según algunos autores, podría empezar a considerarse como una enfermedad quirúrgica intestinal ${ }^{45}$.

\section{BIBLIOGRAFÍA}

1. Whiting DR, Guariguata L, Weil C, et al. IDF diabetes atlas: global estimates of the prevalence of diabetes for 2011 and 2030. Diabetes Res Clin Pract 2011; 94:311-321.

2. Gregg EW, Chen H, Wagenknecht LE, et al. Look AHEAD Research Group. Association of an intensive lifestyle intervention with remission of type 2 diabetes. JAMA 2012; 308:2489-2496.

3. Álvarez MG, Fuentes S, Mociulsky J, y col. Actualización de cirugía bariátrica y cirugía metabólica. Revista de la Sociedad Argentina de Diabetes 2013; 47:69-80.

4. Mingrone G, Panunzi S, De Gaetano A, et al. Bariatric surgery versus conventional medical therapy for type 2 diabetes. $\mathrm{N}$ Engl J Med 2012; 366:1577-1585.

5. Dorman RB, Serrot FJ, Miller CJ, et al. Case matched outcomes in bariatric surgery for treatment of type 2 diabetes in the morbidly obese patient. Ann Surg 2012; 255:287-293.

6. Dixon JB, Zimmet P, Alberti KG, et al. International Diabetes Federation Taskforce on Epidemiology and Prevention, Bariatric Surgery: an IDF statement for obese type 2 diabetes. Surg Obes Relat Dis 2011; 7:433-447.

7. World Health Organization. Definition, diagnosis and classification of diabetes mellitus and its complications. Reports of a Who consultation. Part 1: Diagnosis and classification of diabetes meIlitus. Genève: World Health Organization: 1999.

8. De Fronzo RA. Lilly Lecture:The triumvirate: cell, muscle, liver: a collusion responsible for NIDDM. Diabetes 1988; 37:667-687.
9. Larsen N, Vogensen FK, Van Den Berg FW, et al. Gut microbiota in human adults with type 2 diabetes differs from non-diabetic adults. PLoS ONE 5 2010; 9085.

10. Cone JJ, McCutcheon JE, Roitman MF. Ghrelin acts as an interface between physiological state and phasic dopamine signaling. J. Neurosci 2014; 34: 4905-4913.

11. Inzucchi SE, Bergenstal RM, Buse JB, et al. Management of hyperglycemia in type 2 diabetes: a patient-centered approach: position statement of the American Diabetes Association (ADA) and the European Association for the Study of Diabetes (EASD). Diabetes Care 2012; 35:1364-1379.

12. Stratton IM, Adler Al, Neil HA, et al. Association of glycaemia with macrovascular and microvascular complications of type 2 diabetes (UKPDS 35): prospective observational study. BMJ 2000; 321:405-412.

13. King P, Peacock I, Donnelly $R$, et al. The UK Prospective Diabetes Study (UKPDS): clinical and therapeutic implications for type 2 diabetes. British Journal of Clinical Pharmacology 1999; 48(5):643-648.

14. Gæde $\mathrm{P}$, Vedel $\mathrm{P}$, Parving $\mathrm{H}-\mathrm{H}$, Pedersen $\mathrm{O}$. Intensified multifactorial intervention in patients with type 2 diabetes and microalbuminuria: The Steno Type 2 randomised study. N Engl J Med 2003; 348: 383-93.

15. Knowler WC, Barrett-Connor E, Fowler SE, et al. Diabetes Prevention Program Research Group (DPP). Reduction in the incidence of type 2 diabetes with lifestyle intervention or metformin. N Engl J Med 2002; 346:393-403.

16. Joshua $P$, Cummings T, Cummings D. Minireview: hormonal and metabolic mechanisms of diabetes remission after gastrointestinal surgery. Endocrinology 2009; 150:2518-2525.

17. Cummings DE, Flum DR. Gastrointestinal surgery as a treatment for diabetes. JAMA 2008; 299:341-343.

18. Chambers AP, Smith EP, Begg DP, et al. Regulation of gastric emptying rate and its role in nutrient-induced GLP-1 secretion in rats after vertical sleeve gastrectomy. Am J Physiol Endocrinol Metab 2014; 306: 424-432.

19. Laferrere B, et al. Effect of weight loss by gastric bypass surgery vs hypocaloric diet on glucose and incretin levels in patients with type 2 diabetes. J Clin Endocrinol Metab 2008; 93:2479-2485.

20. Martinussen C, Bojsen-Møller KN, Dirksen C, et al. Immediate enhancement of first-phase insulin secretion and unchanged glucose effectiveness in patients with type 2 diabetes after Roux-en-Y gastric bypass. Am J Physiol Endocrinol Metab 2015; 308:E535-E544.

21. Falken Y, Hellström PM, Holst JJ, Näslund E. Changes in glucose homeostasis after Roux-en-Y gastric bypass surgery for obesity at day 3, 2 months, and 1 year after surgery: role of gut peptides. J Clin Endocrinol Metab 2011; 96:2227-2235.

22. Le Roux CW, et al. Gut hypertrophy after gastric bypass is associated with increased glucagon-like peptide 2 and intestinal crypt cell proliferation. Ann Surg 2010; 252:50-56.

23. Andreelli F, Amouyal C, Magnan C, et al. What can bariatric surgery teach us about the pathophysiology of type 2 diabetes? Diabetes \& Metabolism 2009; 35: 499-507.

24. Romero F, Nicolau J, Flores L, et al. Comparable early changes in gastrointestinal hormones after sleeve gastrectomy and RouxEn-Y gastric bypass surgery for morbidly obese type 2 diabetic subjects. Surg Endosc 2012; 26:2231-2239.

25. Seeley RJ, Chambers AP, Sandoval DA. The role of gut adaptation in the potent effects of multiple bariatric surgeries on obesity and diabetes. Cell Metabolism 2015; 21. 
26. Alfa RW, Park S, Skelly KR, et al. Suppression of insulin production and secretion by a decretin hormone. Cell Metab 2015; 21:323-333.

27. Sandler BJ, Rumbaut $\mathrm{R}$, Swain $\mathrm{CP}$, et al. Human experience with an endoluminal, endoscopic, gastrojejunal bypass sleeve. Surg Endosc 2011; 25:3028-3033.

28. Cavin JB, Couvelard A, Lebtahi R, et al. Differences in alimentary glucose absorption and intestinal disposal of blood glucose after Roux-en-Y gastric bypass vs sleeve gastrectomy. Gastroenterology 2016; 150:454-464.e9.

29. Scarlett JM, Schwartz MW. Gut-brain mechanisms controlling glucose homeostasis. F1000 Prime Rep 2015; 7:12.

30. Depoortere I. Taste receptors of the gut: emerging roles in health and disease. Gut 2014; 63:179-190.

31. Kohli R, Bradley D, Setchell KD, et al. Weight loss induced by Roux-en-Y gastric bypass but not laparoscopic adjustable gastric banding increases circulating bile acids. J Clin Endocrinol Metab 2013; 98: 708-712.

32. Arble D, Sandoval D, Seeley RJ. Mechanisms underlying weight loss and metabolic improvements in rodent models of bariatric surgery. Diabetología 2015; 58:211-220.

33. Li J, Ashrafian $\mathrm{H}$, Bueter M, et al. Metabolic surgery profoundly influences gut microbial-host metabolic cross-talk. Gut 2011; 60:1214-1223.

34. Graessler J, Qin Y, Zhong $H$, et al. Metagenomic sequencing of the human gut microbiome before and after bariatric surgery in obese patients with type 2 diabetes: correlation with inflammatory and metabolic parameters. The Pharmacogenomics Journal 2013; 13: 514-522.

35. Liou AP, Paziuk M, Luevano JM Jr, Machineni S, Turnbaugh PJ, Kaplan LM. Conserved shifts in the gutmicrobiota due to gastric bypass reduce host weight and adiposity. Sci Transl Med 2013; 5:17.

36. Postigo MC, Roca-Rodriguez MM, Camargo A. Lipopolysaccharide and lipopolysaccharide-binding protein levels and their relationship to early metabolic improvement after bariatric surgery. Surgery for Obesity and Related Diseases, 2015.

37. Batterham RL, Cummings DE. Mechanisms of diabetes improvement following bariatric/metabolic surgery. Diabetes Care 2016; 39:893-901.

38. Consenso Argentino de Cirugía Metabólica en pacientes con diabetes tipo 2. Sociedad Argentina de Cirugía de la Obesidad (SACO), Sociedad Argentina de Diabetes (SAD), Sociedad Argentina de Nutrición (SAN), 2014.

39. Consenso Argentino de Cirugía Metabólica. Sociedad Argentina de Cirugía de la Obesidad (SACO), Sociedad Argentina de Diabetes (SAD), Sociedad Argentina de Nutrición (SAN). Revista sociedad Argentina de Diabetes 2015; 49, 3, 95-110.

40. Scopinaro N, Marinari GM, Camerini GB, et al. Specific effects of biliopancreatic diversion on the major components of metabolic syndrome: a long-term follow-up study. Diabetes Care 2005; 28:2406-11.

41. Gloy VL, Briel M, Bhatt DL, et al. Bariatric surgery versus nonsurgical treatment for obesity: a systematic review and metaanalysis of randomised controlled trials. British Medical Journal 2013; 347: 5934.

42. Philip R, Schauer MD, Sangeeta R, et al. Bariatric surgery versus intensive medical therapy in obese patients with diabetes. $\mathrm{N}$ Engl J Med 2012; 366:17.
43. Sjöström $L$, Lindroos AK, Peltonen $M$, et al. Lifestyle, diabetes, and cardiovascular risk factors 10 years after bariatric surgery. $\mathrm{N}$ Engl J Med 2004; 351: 83-93.

44. Sjöström L, Peltonen M, Jacobson P. Association of bariatric surgery with long-term remission of type 2 diabetes and with microvascular and macrovascular complications. JAMA 2014; 311:2297-2304.

45. Sala PC, Torrinhas RS, Heymsfield SB, et al. Type 2 diabetes mellitus: a possible surgically reversible intestinal dysfunction. Obes Surg 2012; 22:167-176.

46. Ribaric G, Buchwald JN, McGlennonTW. Diabetes and weight in comparative studies of bariatric surgery vs conventional medical therapy: a systematic review and meta-analysis. Obes Surg 2014; 24:437-455.

47. Cohen RV, Schiavon CA, Pinheiro JS, et al. Duodenal jejunal bypass for the treatment of type 2 diabetes in patients with body mass index of 22-34 kg/m2. Surg Obes Relat Dis 2007; 3:195-197.

48. Cohen R, Pinheiro JS, Correa JL, et al. Laparoscopic Roux-en-Y gastric bypass for $\mathrm{BMI}<35 \mathrm{~kg} / \mathrm{m}^{2}$ : a tailored approach. Surg Obes Relat Dis 2006; 2:401-404.

49. Sjostrom L. Review of the key results from the Swedish Obese Subjects (SOS) trial. A prospective controlled intervention study of bariatric surgery. J Intern Med 2013; 273:219-234.

50. Carlsson LM, Peltonen M, Ahlin S, et al. Bariatric surgery and prevention of type 2 diabetes in Swedish Obese Subjects. N Engl J Med 2012; 367: 695-704.

51. Sangeeta R, Deepak L, Kathy Wolsky MPH, et al. Metabolic effects of bariatric surgery in patients with moderate obesity and type 2 diabetes. Diabetes Care 2013; 36:2175-2182.

52. Schauer PR, Bhatt DL, Kirwan JP, et al. For the STAMPEDE Investigators. Bariatric surgery versus intensive medical therapy for diabetes 3 year outcomes. N Engl J Med 2014; 370:2002-2013.

53. Nocca D, Krawczykowsky D, Bomans B, et al. Prospective multicenter study of 163 sleeve gastrectomies: results at 1 and 2 years. Obes Surg 2008; 18:560-5.

54. Kehagias I, Karamanakos SN, Argentou M, et al. Randomized clinical trial of laparoscopic Roux-en-Y gastric bypass versus laparoscopic sleeve gastrectomy for the management of patients with $\mathrm{BMI}<50 \mathrm{~kg} / \mathrm{m}^{2}$. Obes Surg 2011; 21:1650-1656.

55. Vetter ML, Ritter S, Wadden TA, et al. Comparison of bariatric surgical procedures for diabetes remission: efficacy and mechanisms. Diabetes spectrum: a publication of the American Diabetes Association 2012; 25(4):200-210.

56. Courcoulas AP, Christian NJ, Belle SH, et al. Weight change and health outcomes at 3 years after bariatric surgery among individuals with severe obesity. JAMA 2013; 310:2416-2425.

57. AdamsTD, Davidson LE, Litwin SE, et al. Health benefits of gastric bypass surgery after 6 years. JAMA 2012; 308:1122-1131.

58. Singh A, Heffron S, Zagzag J, et al. Does bariatric surgery ameliorate the dyslipidemia of obesity? Observations from five years of follow-up after gastric banding. J Am Coll Cardiol 2013; 61: 1388.

59. Brethauer SA, Schauer PR, Romero-Talamás H, et al. Can diabetes be surgically cured? Long term metabolic effects of bariatric surgery in obese patients with type 2 diabetes mellitus. Ann Surg 2013; 258:628-36. 